\title{
OPERATORS OF P-VARIATION AND THE EVOLUTION REPRESENTATION PROBLEM
}

BY

\author{
M. A. FREEDMAN
}

\begin{abstract}
In contrast to a continuous linear semigroup, a continuous linear evolution $U(\cdot)$ may be nondifferentiable or of unbounded variation. In order to study these evolutions we introduce a class of operator-valued functions $A(\cdot)$ which satisfy a generalized bounded variation condition and represent $U$ as the product integral $U=\Pi[I+d A]$.
\end{abstract}

1. Introduction. Let $X$ denote an arbitrary Banach space with norm $|\cdot|$. A family of bounded (linear) operators $U=\{U(t, s)\}_{t \geqslant s}$ on $X$ is called a (linear) evolution on $X$ if $U$ satisfies

$$
\begin{aligned}
& U(t, s) U(s, r)=U(t, r) \quad(t \geqslant s \geqslant r), \\
& U(s, s)=I, \quad \text { the identity operator }(\forall s) .
\end{aligned}
$$

Evolutions arise in analysis in various fundamental ways. Consider, for example, the abstract integral equation

$$
f(t)=x_{a}+\int_{a}^{t} \mathbb{Q}(\tau) f(\tau) d \tau \quad(a \leqslant t \leqslant b),
$$

where for each $t$ in the interval $[a, b], \mathscr{Q}(t)$ is an everywhere defined operator on $X$. If, for each $x_{a} \in X,(1.2)$ should have a unique solution $f(t)$, then $f(t)=U(t, a) x_{a}$, where $U$ satisfies (1.1) for all $a \leqslant s \leqslant t \leqslant b$. Or consider, in the area of stochastic processes, a nonstationary Markov process having countable state space $\left\{e_{1}, e_{2}, \ldots\right\}$. Such a Markov process is specified by its transition probabilities $P_{i j}(t, s)$ and by its initial probability distribution $\Pi_{i}(a)$, where $P_{i j}(t, s)$, for $t \geqslant s \geqslant a$, is the probability the process is in state $e_{i}$ at time $t$ given that at time $s$ the process was in state $e_{j}$, and $\Pi_{i}(a)$ is the probability that at the initial time $t=a$, the process is in state $e_{i}$. If now, we denote the infinite matrix $\left[P_{i j}(t, s)\right]$ by $P(t, s)$, then, by virtue of the Chapman-Kolmogorov equations, $P=\{P(t, s)\}_{t \geqslant s}$ is an evolution. Furthermore, if $\Pi(t)$ is the column vector with components $\Pi_{i}(t)$, then $\Pi(t)=P(t, a) \Pi(a)$.

In both of these examples the state vectors $f(t)$ and $\Pi(t)$ depend on the time $t$, the initial states $x_{a}$ and $\Pi(a)$, and the initial time $t=a$. However, had $\mathbb{Q}(t)$ been constant in time and $P(t, s)$ been a function only of the time difference $t-s$, then in this time homogeneous situation, the state vectors would have depended only on their initial states and the time elapsed. That is, we would have had $f(t)=U(t-a) x_{a}$

Received by the editors May 27, 1982.

1980 Mathematics Subject Classification. Primary 47D05.

Key words and phrases. p-variation, evolution, product integral, Stieltjes integral.

(C) 1983 American Mathematical Society $0002-9939 / 82 / 0000-0887 / \$ 04.50$ 
and $\Pi(t)=P(t-a) \Pi(a)$, where $U$ and $P$ are semigroups; a semigroup $T$ being a family of bounded operators $T=\{T(t)\}_{t \geqslant 0}$ which satisfy

$$
T(t+s)=T(t) T(s), \quad T(0)=I .
$$

Therefore, the evolution property (1.1) might well be thought of as the nonhomogeneous generalization of the semigroup property (1.3).

The problem of determining all functions which satisfy (1.3) was initiated by Cauchy when, in his "Cours d'Analyse" [1], he proved that every real-valued, continuous function $T(t)$ satisfying (1.3) is necessarily an exponential function, $e^{\alpha t}$, $\alpha \in \mathbf{R}$. Since then this problem has been extensively researched by many mathematicians, including J. von Neumann, M. H. Stone, D. S. Nathan, E. Hille, N. Dunford, K. Yosida, R. S. Phillips, W. Feller and M. Crandall. In Nathan's work [11], it was shown in the general setting, where $T(\cdot)$ is $X$-operator-valued and uniformly continuous, that there exists a bounded operator on $X, \Theta$, known as the infinitesimal generator of $T$, such that $T(t)=e^{\mathfrak{Q} t}$. Thus $T$, in this case, is differentiable with $d^{+} T(0) / d t$ equal to its infinitesimal generator. An evolution, however, may be continuous without being differentiable. (Consider the evolution $e^{(g(t)-g(s))}$ where $g$ is continuous and nowhere differentiable.) In fact, evolutions may exhibit a variety of pathologies-some of which will be discussed below. Therefore, the problem of characterizing all operator-valued functions which satisfy the evolution equation (1.1) is formidable and, until recently, has been largely neglected in the literature.

In order to motivate our general approach to the study of evolutions, let us first consider the special type of evolution $U$ formed as $U(t, s)=T(t-s), t \geqslant s$, where $T$ is a uniformly continuous semigroup. Suppose $T$ has infinitesimal generator $\mathcal{Q}$ ? Then we may write $U(t, s)=e^{d A(t, s)}$, where $d A(t, s)=A(t)-A(s)$ and $A(t)=t(\mathcal{Q}$. Furthermore, it is easily verified that $d A(t, s)$ can be expressed as the sum integral $\Sigma_{s}^{t}[U-I]$, defined as the limit of successive refinements of partitions $s=\sigma_{0}<\sigma_{1}<$ $\cdots<\sigma_{n}=t$ of $[s, t]$ of the finite sum $\sum_{i=1}^{n}\left[U\left(\sigma_{i}, \sigma_{i-1}\right)-I\right]$. Moreover, $U$ can be expressed as the product integral of its additive generator $d A$ as $U(t, s)=\prod_{s}^{t}[I+d A]$, where the product integral is defined as the limit of finite products over successive refinements of partitions. With this example in mind, we propose the

Evolution representation problem. Given an evolution $U$ on a Banach space $X$, determine a family of operators $A=\{A(s)\}_{s \in \mathbf{R}}$ on $X$ and a function $\mathscr{F}$ defined on $d A=\{d A(t, s)\}_{t \geqslant s}$ such that the product integral $\Pi_{s}^{t} \mathscr{F}(d A)$ exists and equals $U(t, s)$.

We shall be considering the case where $U(\cdot)$ is uniformly continuous and work with $\mathscr{F}(d A)=I+d A$. In [7], where $U$ is strongly continuous, $\mathscr{F}(d A)=[I-d A]^{-1}$ is used successfully and in [5], which deals with discontinuous evolutions,

$$
\mathscr{F}(d A)(t, s)=\left[I+d A\left(t, t^{-}\right)\right]\left[I-d A\left(t^{-}, s^{+}\right)\right]^{-1}\left[I+d A\left(s^{+}, s\right)\right]
$$

is used. In both of these papers the family of operators $d A$ is given by $d A(t, s)=$ $\Sigma_{s}^{t}[U-I]$ and serves as the additive generator of $U$ in the sense that $U=\Pi \mathscr{F}(d A)$. This idea of pairing multiplicative evolutions with additive generators is originally due to J. S. Mac Nerney [9, 10]. In effect, Mac Nerney was first to construct a noncommutative logarithm $d A=\Sigma[U-I]$, of the noncommutative family $\{U(t, s)\}_{t \geqslant s}$, to act as the inverse of the noncommutative exponential $\Pi[I+d A]$. 
Mac Nerney was concerned with evolutions and generators of norm bounded variation. For the one-dimensional evolution $u(t, s)=e^{d a(t, s)}$, where $a$ is a realvalued function, this is tantamount to requiring that $a$ be monotone. Suppose, however, continuity is substituted for monotonicity of $a$. Then the commutativity of $\{u(t, s)\}_{t \geqslant s}$ and $\{d a(t, s)\}_{t>s}$ allows: $d a(t, s)=\ln u(t, s)$ and $u(t, s)=e^{d a(t, s)}$. Yet these representations can be achieved without appealing to commutativity. First we write

$$
d a(t, s)=\sum_{s}^{t}\left[e^{d a}-1\right]-\sum_{s}^{t}\left[\frac{(d a)^{2}}{2 !}+\frac{(d a)^{3}}{3 !}+\cdots\right] .
$$

Hence if $d a$ should satisfy $\Sigma_{s}^{t}(d a)^{2}=0$, it follows that $d a(t, s)=\Sigma_{s}^{t}[u-1]$. Furthermore, $u(t, s)=\prod_{s}^{t}[1+d a]$, for if $\tau=\left\{\tau_{i}\right\}_{0}^{n}$ is a partition of $[s, t]$ then

$$
\begin{aligned}
\mid u(t, s) & -\prod_{i=1}^{\tau}[1+d a] \mid \\
= & \left|\sum_{i=1}^{n} \prod_{j=i+1}^{n}\left[1+d a\left(\tau_{j}, \tau_{j-1}\right)\right]\left\{e^{d a\left(\tau_{i}, \tau_{i-1}\right)}-1-d a\left(\tau_{i}, \tau_{i-1}\right)\right\} \prod_{j=1}^{i-1} e^{d a\left(\tau, \tau_{j-1}\right)}\right| \\
& \leqslant k \sum_{i=1}^{n}\left\{\frac{\left|d a\left(\tau_{i}, \tau_{i-1}\right)\right|^{2}}{2 !}+\frac{\left|d a\left(\tau_{i}, \tau_{i-1}\right)\right|^{3}}{3 !}+\cdots\right\},
\end{aligned}
$$

which approaches zero as $\tau$ becomes ever finer. Thus in this one-dimensional continuous case the condition $\Sigma_{s}^{t}|d a|^{2}=0, t \geqslant s$, is sufficient for our purposes. In the two-dimensional case where $A(\cdot)$ is a $2 \times 2$ matrix operator, this quadratic condition plus a dissipative condition was found to be sufficient for the existence of $\Pi[I+d A]$. But in the case of an infinite-dimensional operator $A(\cdot)$, it was found necessary to seek a more tractable condition.

Definition 1.1. Given $a<b$ and $0<p$, let $f$ be a function from $[a, b]$ to a normed vector space $(Z,|\cdot|)$. The $p$-variation of $f$ over $[a, b], V_{p}(f ; b, a)$, is defined to be the supremum of the quantities

$$
\left[\sum_{\tau}|d f|^{p}\right]^{1 / p}=\left[\sum_{i=1}^{n}\left|f\left(\tau_{i}\right)-f\left(\tau_{i-1}\right)\right|^{p}\right]^{1 / p}
$$

over all partitions $\tau=\left\{\tau_{i}\right\}_{0}^{n}$ of $[a, b]$.

Definition 1.2. When $Z=X$, let $W_{p}([a, b])$ denote the set of all continuous $X$-valued functions $h$ on $[a, b]$ for which $V_{p}(h ; b, a)$ is finite.

Definition 1.3. When $Z=\mathscr{B} \mathcal{L}(X)$, the bounded linear operators $L$ on $X$, let $|\cdot|$ denote the operator norm $|L|=\sup \{|L x| /|x|: x \in X,|x| \neq 0\}$. Define $\mathcal{Q S}_{p}([a, b])$ to be the set of all maps $A:[a, b] \rightarrow \mathscr{R}(X)$ which are uniformly continuous (i.e. continuous in the operator-norm topology) and such that $V_{p}(A ; b, a)<\infty$.

REMARKs 1.1. (i) By expressing $\left|d A\left(\tau_{i}, \tau_{i-1}\right)\right|^{2}$ as $\left|d A\left(\tau_{i}, \tau_{i-1}\right)\right|^{2-p}\left|d A\left(\tau_{i}, \tau_{i-1}\right)\right|^{p}$, we see that if $0<p<2$ and $A \in \mathcal{W}_{p}([a, b])$, then $\Sigma_{s}^{t}|d A|^{2}=0$. Therefore, for our purposes we shall restrict $p$ to the interval $(0,2)$.

(ii) $\mathscr{W}_{p}([a, b])$ with $p=1$ is exactly the set of continuous functions of norm bounded variation on $[a, b]$. It was this $p=1$ condition that Mac Nerney relied on in $[9,10]$ (though Mac Nerney did not assume continuity). Likewise, subsequent 
papers in which an evolution is represented as the product integral of a generator (e.g. $[5,7])$, are restricted to functions of either norm or strong bounded variation. The analysis we attempt here for $1<p<2$ contrasts with the $p=1$ case. For one thing, vector-valued functions of bounded variation can be bounded above in norm by increasing functions. This permits much of the analysis to be performed outside the original Banach space and in the space of increasing functions. No such convenience is available when $p>1$. Moreover, as should become evident as we proceed, we shall be denied the use of the triangle inequality many times when its application would seem necessary.

(iii) The notion of $p$-variation of a real-valued function $f$ first appeared in Wiener's paper [12]. Since then, these functions have been studied vigorously in a series of papers, a sampling of which is $[\mathbf{2}, \mathbf{6}, \mathbf{8}, \mathbf{1 3}$ ] (see also M. Bruneau's tract [3].) Apparently the case where $f$ is vector-valued has previously not been considered.

(iv) The realization that several key results in L. C. Young's paper [13] could be extended to a Banach space setting has been instrumental in the development of the present work. For the sake of completeness, the proofs of these extended results shall be included in $\S 3$.

In [4] it was shown that in sup-norm $\mathbf{R}^{n}$, accretiveness and continuity of generators, or contractiveness and continuity of evolutions, suffice to imply bounded variation. Yet with other norms on $\mathbf{R}^{n}$, for example the Euclidean norm, it is possible for a continuous, contractive evolution $U$ to be generated by a continuous, accretive generator $d A$, even though neither $U-I$ nor $d A$ are of bounded variation (see [4, p. 573]). Even in the extension of $\mathbf{R}^{n}$ to $l_{\infty}(\mathbf{R})$, the author has found a norm continuous, contractive evolution $U$ having a norm continuous, accretive generator $d A$ with neither $U-I$ nor $d A$ of norm or strong bounded variation. Therefore, the possibility of carrying out a program of matching continuous, contractive evolutions with continuous, accretive generators is enhanced for a greater variety of normed spaces by the present theory over the bounded variation based theories.

2. Properties of the $p$-variation. As discussed in the previous section, our natural domain of interest is $p \in(0,2)$, and unless otherwise stated, the reader should assume that $p$ is so restricted. Though surely Lemma 2.2 below is known, Lemma 2.3 appears to be new. First we present a simple but useful inequality.

LeMma 2.1. For $L, M \geqslant 0$ and $0 \leqslant p \leqslant 2:(L+M)^{p} \leqslant L^{p}+M^{p}+2(L M)^{p / 2}$.

Proof. Setting $r=p / 2$, it suffices to prove that $L+M \leqslant\left(L^{r}+M^{r}\right)^{1 / r}$. But

$$
\frac{L+M}{\left(L^{r}+M^{r}\right)^{1 / r}}=\left(\frac{L^{r}}{L^{r}+M^{r}}\right)^{1 / r}+\left(\frac{M^{r}}{L^{r}+M^{r}}\right)^{1 / r} \leqslant \frac{L^{r}}{L^{r}+M^{r}}+\frac{M^{r}}{L^{r}+M^{r}}=1 .
$$

LEMMA 2.2. If $A$ is in $\mho_{p}([a, b])$ and $c$ is in $[a, b)$, then $V_{p}(A ; \xi, c) \downarrow 0$ as $\xi \downarrow c$.

Proof. It follows immediately from Definition 1.1 that for all $\mu \in(c, \xi)$,

$$
V_{p}^{p}(A ; \xi, \mu)+V_{p}^{p}(A ; \mu, c) \leqslant V_{p}^{p}(A ; \xi, c) .
$$


Given $\varepsilon>0$, let $\delta>0$ be such that $|d A(t, s)|^{p}<\varepsilon / 2$ whenever $0 \leqslant t-s<\delta$, and let $\tau=\left\{\tau_{i}\right\}_{0}^{n}$ be a partition of $[c, b]$ such that

$$
V_{p}^{p}(A ; b, c)-\frac{\varepsilon}{2} \leqslant \sum_{\tau}|d A|^{p} .
$$

Suppose $\tau_{1}<c+\delta$. Then

$$
\sum_{\tau}|d A|^{p}=\left|d A\left(\tau_{1}, c\right)\right|^{p}+\sum_{i=2}^{n}\left|d A\left(\tau_{i}, \tau_{i-1}\right)\right|^{p}<\frac{\varepsilon}{2}+V_{p}^{p}\left(A ; b, \tau_{1}\right) .
$$

Hence, using (2.1)-(2.3),

$$
\begin{aligned}
V_{p}^{p}\left(A ; \tau_{1}, c\right) & \leqslant V_{p}^{p}(A ; b, c)-V_{p}^{p}\left(A ; b, \tau_{1}\right) \\
& <\frac{\varepsilon}{2}+\sum_{\tau}|d A|^{p}+\frac{\varepsilon}{2}-\sum_{\tau}|d A|^{p}=\varepsilon,
\end{aligned}
$$

and we are done.

Now suppose $\tau_{1} \geqslant c+\delta$. Then, making use of Lemma 2.1,

$$
\begin{aligned}
\sum_{\tau}|d A|^{p} \leqslant & |d A(c+\delta, c)|^{p}+\left|d A\left(\tau_{1}, c+\delta\right)\right|^{p} \\
& +2|d A(c+\delta, c)|^{p / 2}\left|d A\left(\tau_{1}, c+\delta\right)\right|^{p / 2}+\sum_{i=2}^{n}\left|d A\left(\tau_{i}, \tau_{i-1}\right)\right|^{p} \\
\leqslant & \varepsilon / 2+2 \sqrt{\varepsilon / 2} \alpha^{p / 2}+V_{p}^{p}(A ; b, c+\delta),
\end{aligned}
$$

where $\alpha=\max _{a \leqslant s \leqslant t \leqslant b}|d A(t, s)|$. Therefore

$$
V_{p}^{p}(A ; c+\delta, c) \leqslant V_{p}^{p}(A ; b, c)-V_{p}^{p}(A ; b, c+\delta) \leqslant \varepsilon / 2+2 \alpha^{p / 2} \sqrt{\varepsilon / 2} .
$$

Lemma 2.3. Let $A \in \mathcal{Q U}_{p}([a, b])$. For all $a \leqslant \mu<\eta<\nu \leqslant b$,

$$
V_{p}^{p / 2}(A ; \nu, \mu) \leqslant V_{p}^{p / 2}(A ; \nu, \eta)+V_{p}^{p / 2}(A ; \eta, \mu) .
$$

REMARK 2.1. The subadditivity exhibited by the $p$-variation in (2.4) should be compared with the superadditivity exhibited in inequality (2.1).

Proof. Let $\tau=\left\{\tau_{i}\right\}_{0}^{n}$ be an arbitrary partition of $[\mu, \nu]$. If the point $\eta$ should be in $\tau$, say $\eta=\tau_{r}$ for some $r \in[0, n]$, then

$$
\begin{aligned}
\sum_{\tau}|d A|^{p} & =\sum_{i=1}^{r}\left|d A\left(\tau_{i}, \tau_{i-1}\right)\right|^{p}+\sum_{i=r+1}^{n}\left|d A\left(\tau_{i}, \tau_{i-1}\right)\right|^{p} \\
& \leqslant V_{p}^{p}(A ; \eta, \mu)+V_{p}^{p}(A ; \nu, \eta) .
\end{aligned}
$$

On the other hand, if $\eta \notin \tau$ then $\eta \in\left(\tau_{r-1}, \tau_{r}\right)$ for some $r \in[1, n]$. Hence,

$$
\begin{aligned}
\sum_{\tau}|d A|^{p} \leqslant & \sum_{i=1}^{r-1}\left|d A\left(\tau_{i}, \tau_{i-1}\right)\right|^{p}+\left|d A\left(\eta, \tau_{r-1}\right)\right|^{p} \\
& +2\left|d A\left(\tau_{r}, \eta\right)\right|^{p / 2}\left|d A\left(\eta, \tau_{r-1}\right)\right|^{p / 2} \\
& +\left|d A\left(\tau_{r}, \eta\right)\right|^{p}+\sum_{i=r+1}^{n}\left|d A\left(\tau_{i}, \tau_{i-1}\right)\right|^{p} \\
\leqslant & V_{p}^{p}(A ; \eta, \mu)+2 V_{p}^{p / 2}(A ; \nu, \eta) V_{p}^{p / 2}(A ; \eta, \mu)+V_{p}^{p}(A ; \nu, \eta) .
\end{aligned}
$$


In either case we have

$$
\sum_{\tau}|d A|^{p} \leqslant\left[V_{p}^{p / 2}(A ; \nu, \eta)+V_{p}^{p / 2}(A ; \eta, \mu)\right]^{2}
$$

which implies (2.4).

Lemma 2.4. Let $A \in \mathcal{O}_{p}([a, b])$. Then $V_{p}(A ; \cdot)$ is continuous on the triangle $\Delta=\{(t, s): b \geqslant t \geqslant s \geqslant a\}$.

Proof. For $\left(t_{0}, s_{0}\right) \in \Delta$, suppose $(v, u) \in \Delta$ is such that $v \geqslant t_{0} \geqslant u \geqslant s_{0}$. Then

$$
\begin{aligned}
\left|V_{p}^{p / 2}\left(A ; t_{0}, s_{0}\right)-V_{p}^{p / 2}(A ; v, u)\right| \leqslant & \left|V_{p}^{p / 2}\left(A ; t_{0}, s_{0}\right)-V_{p}^{p / 2}\left(A ; t_{0}, u\right)\right| \\
& +\left|V_{p}^{p / 2}\left(A ; t_{0}, u\right)-V_{p}^{p / 2}(A ; v, u)\right|,
\end{aligned}
$$

which, by Lemma 2.3 , is bounded above by $V_{p}^{p / 2}\left(A ; u, s_{0}\right)+V_{p}^{p / 2}\left(A ; v, t_{0}\right)$. Likewise, for other possible orderings of $t_{0}, s_{0}, u$ and $v$, we obtain similar type upper bounds, each of which, by Lemma 2.2, approaches zero as $(v, u)$ approaches $\left(t_{0}, s_{0}\right)$.

ReMarK 2.2. Since $V_{p}(A ; \xi, \xi)$ is identically zero for $\xi \in[a, b]$, it follows that given $\varepsilon>0$, there exists $\delta_{0}>0$ which makes $V_{p}(A ; \xi+\delta, \xi)<\varepsilon$ for all $\xi \in[a, b)$ and all $\delta \in\left[0, \delta_{0}\right]$ such that $\xi+\delta \in[a, b]$.

3. Existence of the Stieltjes integral. We show that if $A \in \mathcal{W}_{p}([a, b])$ and $f \in$ $W_{p}([a, b])$ for some fixed $0<p<2$, then the Stieltjes integral $\int_{a}^{\xi} d A f$ exists and, as a function of $\xi$, belongs to $W_{p}([a, b])$. The methods used in this section are essentially taken from L. C. Young's beautiful paper [13].

LEMmA 3.1. Given positive numbers $\rho_{i}, i=1, \ldots, n$, there exists $k \in[1, n]$ such that

$$
\rho_{k} \leqslant\left[\frac{1}{n} \sum_{i=1}^{n} \rho_{i}^{p}\right]^{1 / p}
$$

Proof. The lemma is an easy consequence of the fact that the geometric mean $\left[\rho_{1}^{p} \rho_{2}^{p} \cdots \rho_{n}^{p}\right]^{1 / n}$ does not exceed the arithmetic mean $n^{-1} \sum_{i=1}^{n} \rho_{i}^{p}$.

Given a sequence $R=\left\{R_{i}\right\}_{1}^{n}$ drawn from $X$ or $\beta \mathcal{L}(X)$, for each integer $k \in[1, n$ - 1] we define the sequence operator $\mathscr{P}_{k}$ acting on $R$ to be the $n-1$ element sequence $\mathscr{P}_{k} R=\left(R_{1}, R_{2}, \ldots, R_{k}+R_{k+1}, R_{k+2}, \ldots, R_{n}\right)$. We further define $\mathcal{S}_{p}(R)$ to be the largest value of $\left[\sum_{i=1}^{m}\left|Y_{i}\right|^{p}\right]^{1 / p}$ such that $\left(Y_{1}, \ldots, Y_{m}\right)$, with $m \leqslant n$, is the result of a composition of sequence operators $\mathscr{P}_{k}$ acting on $R$.

REMARK 3.1. In the case where $A \in \mathcal{W}_{p}([a, b]), \tau$ is a partition of the subinterval $[\mu, \nu] \subseteq[a, b]$, and $R$ is the sequence given by $R_{i}=d A\left(\tau_{i}, \tau_{i-1}\right)$, we see that $\delta_{p}(R) \leqslant V_{p}(A ; \nu, \mu)$.

LEMMA 3.2. Given a sequence $S=\left\{S_{i}\right\}_{1}^{n} \subseteq \mathscr{B} \mathscr{L}(X)$ and a sequence $h=\left\{h_{i}\right\}_{1}^{n}$ taken from $X$ or $\mathscr{B} \mathcal{L}(X)$,

$$
\left|\sum_{1 \leqslant i \leqslant j \leqslant n} S_{i} h_{j}\right| \leqslant\left[1+\zeta\left(\frac{2}{p}\right)\right] \delta_{p}(S) \delta_{p}(h),
$$

where $\zeta(\cdot)$ denotes the Riemann zeta function. 
Proof. For any integer $k \in[1, n-1]$, if $Y=\mathscr{P}_{k} S$ and $x=\mathscr{P}_{k} h$ then

$$
Y_{r}, x_{r}= \begin{cases}S_{r}, h_{r} & \text { if } r<k \\ S_{r}+S_{r+1}, h_{r}+h_{r+1} & \text { if } r=k, \\ S_{r+1}, h_{r+1} & \text { if } k<r \leqslant n-1\end{cases}
$$

Therefore,

$$
\begin{aligned}
\sum_{1 \leqslant j \leqslant n-1} & \left(Y_{1}+\cdots+Y_{j}\right) x_{j}=\sum_{1 \leqslant j<k}\left(S_{1}+\cdots+S_{j}\right) h_{j} \\
& +\left(S_{1}+\cdots+S_{k+1}\right)\left(h_{k}+h_{k+1}\right)+\sum_{k+1 \leqslant j \leqslant n-1}\left(S_{1}+\cdots+S_{j+1}\right) h_{j+1} \\
= & S_{k+1} h_{k}+\sum_{1 \leqslant j \leqslant n}\left(S_{1}+\cdots+S_{j}\right) h_{j} .
\end{aligned}
$$

Now by Lemma 3.1, for some integer $k \in[1, n-1]$, we have

$$
\begin{aligned}
\left|\sum_{1 \leqslant i \leqslant j \leqslant n} S_{i} h_{j}\right| & \leqslant\left|S_{k+1} h_{k}\right|+\left|\sum_{1 \leqslant i \leqslant j \leqslant n-1} Y_{i} x_{j}\right| \\
& \leqslant\left[\frac{1}{n-1} \sum_{i=1}^{n-1}\left|S_{i+1}\right|^{p}\right]^{1 / p}\left[\frac{1}{n-1} \sum_{i=1}^{n-1}\left|h_{i}\right|^{p}\right]^{1 / p}+\left|\sum_{1 \leqslant i \leqslant j \leqslant n-1} Y_{i} x_{j}\right| \\
& \leqslant(n-1)^{-2 / p} S_{p}(S) S_{p}(h)+\left|\sum_{1 \leqslant i \leqslant j-1} Y_{i} x_{j}\right| .
\end{aligned}
$$

Similar reasoning applied to the sequences $Y$ and $x$ yields

$$
\left|\sum_{1 \leqslant i \leqslant j \leqslant n-1} Y_{i} x_{j}\right| \leqslant(n-2)^{-2 / p} \mathcal{S}_{p}(Y) \mathcal{S}_{p}(x)+\left|\sum_{1 \leqslant i \leqslant j \leqslant n-2} Z_{i} y_{j}\right|
$$

where $Z=\mathscr{P}_{k} Y$ and $y=\mathscr{P}_{k} x$ for some index $k \in[1, n-2]$. Furthermore, we see that $\varsigma_{p}(Y) \leqslant \delta_{p}(S)$ and $\delta_{p}(x) \leqslant \delta_{p}(h)$. Continuing in this way thus yields

$$
\begin{aligned}
\left|\sum_{1 \leqslant i \leqslant j \leqslant n} S_{i} h_{j}\right| & \leqslant\left[\left\{(n-1)^{-2 / p}+(n-2)^{-2 / p}+\cdots+1\right\}+1\right] \mathcal{\delta}_{p}(S) \delta_{p}(h) \\
& \leqslant\left[\sum_{n=1}^{\infty} n^{-2 / p}+1\right] \delta_{p}(h),
\end{aligned}
$$

which is the desired result.

We should also record that the related inequality

$$
\left|\sum_{1 \leqslant j \leqslant i \leqslant n} S_{i} h_{j}\right| \leqslant\left[1+\zeta\left(\frac{2}{p}\right)\right] \delta_{p}(S) \delta_{p}(h)
$$

also holds true.

Lemma 3.3. Given $A \in \mathcal{W}_{p}([a, b])$ and $f \in W_{p}([a, b])$, for each $\eta \leqslant \xi \in[a, b]$ the Stieltjes integral $\int_{\eta}^{\xi} d A f$ exists and satisfies, for any $\theta \in[\eta, \xi]$,

$$
\left|\int_{\eta}^{\xi} d A f-[A(\xi)-A(\eta)] f(\theta)\right| \leqslant 2\left[2+\zeta\left(\frac{2}{p}\right)\right] V_{p}(A ; \xi, \eta) V_{p}(f ; \xi, \eta)
$$


Proof. The Stieltjes integral $\int_{\eta}^{\xi} d A f$ may be viewed as the sum integral

$$
\sum_{\substack{\eta \\ s \leqslant \sigma}}^{\xi} d A(t, s) f(\sigma)
$$

and hence its existence is defined in terms of successive refinements of partitions of $[\eta, \xi]$, as discussed in $\S 1$. However, we shall prove existence in the more exacting Riemann sense: given $\varepsilon>0$ there exists $\delta>0$ such that for every partition $\tau=\left\{\tau_{i}\right\}_{0}^{n}$ of $[\eta, \xi]$ of length $|\tau|=\max _{1 \leqslant i \leqslant n}\left|\tau_{i}-\tau_{i-1}\right|<\delta$ and every choice of intermediary points $\sigma_{i} \in\left[\tau_{i-1}, \tau_{i}\right]$, the sum $\sum_{i=1}^{n} d A\left(\tau_{i}, \tau_{i-1}\right) f\left(\sigma_{i}\right)$ differs by at most $\varepsilon$ in norm from the integral.

Now given $\mu \leqslant \nu \in[a, b]$, let $\lambda=\left\{\lambda_{i}\right\}_{0}^{N}$ be a partition of $[\mu, \nu]$. From Remark 3.1 and Lemma 3.2 we see that

$$
\begin{aligned}
\left|\sum_{i=1}^{N} d A\left(\lambda_{i}, \lambda_{i-1}\right) f\left(\lambda_{i-1}\right)-[A(\nu)-A(\mu)] f(\nu)\right| \\
\quad=\left|\sum_{1 \leqslant i \leqslant j \leqslant N} d A\left(\lambda_{i}, \lambda_{i-1}\right) d f\left(\lambda_{j}, \lambda_{j-1}\right)\right| \leqslant\left[1+\zeta\left(\frac{2}{p}\right)\right] V_{p}(A ; \nu, \mu) V_{p}(f ; \nu, \mu) .
\end{aligned}
$$

As well, we have

$$
\begin{aligned}
\left|\sum_{i=1}^{N} d A\left(\lambda_{i}, \lambda_{i-1}\right) f\left(\lambda_{i-1}\right)-[A(\nu)-A(\mu)] f(\mu)\right| \\
\quad=\left|\sum_{1 \leqslant j \leqslant i<N} d A\left(\lambda_{i}, \lambda_{i-1}\right) d f\left(\lambda_{j}, \lambda_{j-1}\right)-\sum_{i=1}^{N} d A\left(\lambda_{i}, \lambda_{i-1}\right) d f\left(\lambda_{i}, \lambda_{i-1}\right)\right|,
\end{aligned}
$$

which, by equation (3.1) and Hölder's inequality, is no greater than $[2+\zeta(2 / p)] V_{p}(A ; \nu, \mu) V_{p}(f ; \nu, \mu)$. Hence given any point $\lambda_{r}$ in the partition $\lambda$ :

$$
\begin{aligned}
\left|\sum_{i=1}^{N} d A\left(\lambda_{i}, \lambda_{i-1}\right) f\left(\lambda_{i-1}\right)-[A(\nu)-A(\mu)] f\left(\lambda_{r}\right)\right| \\
\leqslant\left|\sum_{i=1}^{r} d A\left(\lambda_{i}, \lambda_{i-1}\right) f\left(\lambda_{i-1}\right)-\left[A\left(\lambda_{r}\right)-A(\mu)\right] f\left(\lambda_{r}\right)\right| \\
\quad+\left|\sum_{i=r+1}^{N} d A\left(\lambda_{i}, \lambda_{i-1}\right) f\left(\lambda_{i-1}\right)-\left[A(\nu)-A\left(\lambda_{r}\right)\right] f\left(\lambda_{r}\right)\right| \\
\leqslant[2+\zeta(2 / p)]\left\{V_{p}\left(A ; \lambda_{r}, \mu\right) V_{p}\left(f ; \lambda_{r}, \mu\right)+V_{p}\left(A ; \nu, \lambda_{r}\right) V_{p}\left(f ; \nu, \lambda_{r}\right)\right\} \\
\leqslant 2[2+\zeta(2 / p)] V_{p}(A ; \nu, \mu) V_{p}(f ; \nu, \mu) .
\end{aligned}
$$

If now $\left\{\tau_{i}\right\}_{0}^{n}$ and $\left\{\tau_{i}^{\prime}\right\}_{0}^{n^{\prime}}$ are any two partitions of $[\eta, \xi]$ with intermediary points $\sigma_{i} \in\left[\tau_{i-1}, \tau_{i}\right]$ and $\sigma_{i}^{\prime} \in\left[\tau_{i-1}^{\prime}, \tau_{i}^{\prime}\right]$, there exists a common refinement containing both partitions and both sets of intermediary points. Hence, by (3.3), it follows that 


$$
\begin{aligned}
& \left|\sum_{i=1}^{n} d A\left(\tau_{i}, \tau_{i-1}\right) f\left(\sigma_{i}\right)-\sum_{i=1}^{n^{\prime}} d A\left(\tau_{i}^{\prime}, \tau_{i-1}^{\prime}\right) f\left(\sigma_{i}^{\prime}\right)\right| \\
& \leqslant 2\left[2+\zeta\left(\frac{2}{p}\right)\right]\left\{\sum_{i=1}^{n} V_{p}\left(A ; \tau_{i}, \tau_{i-1}\right) V_{p}\left(f ; \tau_{i}, \tau_{i-1}\right)\right. \\
& \left.+\sum_{i=1}^{n^{\prime}} V_{p}\left(A ; \tau_{i}^{\prime}, \tau_{i-1}^{\prime}\right) V_{p}\left(f ; \tau_{i}^{\prime}, \tau_{i-1}^{\prime}\right)\right\} \\
& \leqslant\left[2+\zeta\left(\frac{2}{p}\right)\right]\left\{\sum_{i=1}^{n}\left(V_{p}^{2}\left(A ; \tau_{i}, \tau_{i-1}\right)+V_{p}^{2}\left(f ; \tau_{i}, \tau_{i-1}\right)\right)\right. \\
& \left.+\sum_{i=1}^{n^{\prime}}\left(V_{p}^{2}\left(A ; \tau_{i}^{\prime}, \tau_{i-1}^{\prime}\right)+V_{p}^{2}\left(f, \tau_{i}^{\prime}, \tau_{i-1}^{\prime}\right)\right)\right\} \\
& \leqslant\left[2+\zeta\left(\frac{2}{p}\right)\right] \mathscr{R}\left(\tau, \tau^{\prime}\right)\left\{\sum_{i=1}^{n}\left(V_{p}^{p}\left(A ; \tau_{i}, \tau_{i-1}\right)+V_{p}^{p}\left(f ; \tau_{i}, \tau_{i-1}\right)\right)\right. \\
& \left.+\sum_{i=1}^{n^{\prime}}\left(V_{p}^{p}\left(A ; \tau_{i}^{\prime}, \tau_{i-1}^{\prime}\right)+V_{p}^{p}\left(f ; \tau_{i}^{\prime}, \tau_{i-1}^{\prime}\right)\right)\right\},
\end{aligned}
$$

where

$$
\begin{aligned}
\mathscr{T}\left(\tau, \tau^{\prime}\right)=\max _{\substack{1 \leqslant i \leqslant n \\
1 \leqslant j \leqslant n}}\left(V_{p}^{2-p}\left(A ; \tau_{i}, \tau_{i-1}\right), V_{p}^{2-p}\left(f ; \tau_{i}, \tau_{i-1}\right),\right. \\
\\
\left.\quad V_{p}^{2-p}\left(A ; \tau_{j}^{\prime}, \tau_{j-1}^{\prime}\right), V_{p}^{2-p}\left(f ; \tau_{j}^{\prime}, \tau_{j-1}^{\prime}\right)\right) .
\end{aligned}
$$

By Lemma 2.4, given $\varepsilon>0$ there exists $\delta>0$ such that if $|\tau|$ and $\left|\tau^{\prime}\right|$ are each less than $\delta$ then $\Re\left(\tau, \tau^{\prime}\right)$ will be less than $\varepsilon /\left\{2[2+\zeta(2 / p)]\left(V_{p}^{p}(A ; \xi, \eta)+V_{p}^{p}(f ; \xi, \eta)\right)\right\}$. Superadditivity of $V_{p}^{p}(f ; \cdot)$ and $V_{p}^{p}(A ; \cdot)$ applied to (3.4) thus gives the existence of $\int_{\eta}^{\xi} d A f$, and (3.3) now yields inequality (3.2).

Finally, we argue that $\int_{a}^{(\cdot)} d A f \in W_{p}([a, b])$. The continuity of $\int_{a}^{(\cdot)} d A f$ follows easily from (3.2). To show that $V_{p}\left(\int_{a}^{(\cdot)} d A f ; b, a\right)<\infty$, let $\tau=\left\{\tau_{i}\right\}_{0}^{n}$ be a partition of $[a, b]$. Then

$$
\begin{aligned}
{\left[\sum_{i=1}^{n}\left|\int_{\tau_{i-1}}^{\tau_{i}} d A f\right|^{p}\right]^{1 / p} \leqslant } & {\left[\sum_{i=1}^{n}\left|\int_{\tau_{i-1}}^{\tau_{i}} d A f-\left[A\left(\tau_{i}\right)-A\left(\tau_{i-1}\right)\right] f\left(\tau_{i-1}\right)\right|^{p}\right]^{1 / p} } \\
& +\left[\sum_{i=1}^{n}\left|d A\left(\tau_{i}, \tau_{i-1}\right)\right|^{p}\left|f\left(\tau_{i-1}\right)\right|^{p}\right]^{1 / p} \\
\leqslant & 2\left[2+\zeta\left(\frac{2}{p}\right)\right]\left[\sum_{i=1}^{n} V_{p}^{p}\left(A ; \tau_{i}, \tau_{i-1}\right) V_{p}^{p}\left(f ; \tau_{i}, \tau_{i-1}\right)\right]^{1 / p} \\
& +\max _{a \leqslant \xi \leqslant b}|f(\xi)|\left[\sum_{i=1}^{n}\left|d A\left(\tau_{i}, \tau_{i-1}\right)\right|^{p}\right]^{1 / p}
\end{aligned}
$$


Hence, we have the bound

$$
\begin{aligned}
V_{p}\left(\int_{a}^{(\cdot)} d A f ; b, a\right) \leqslant & 2\left[2+\zeta\left(\frac{2}{p}\right)\right] V_{p}(A ; b, a) V_{p}(f ; b, a) \\
& +\max _{a \leqslant \xi \leqslant b}|f(\xi)| V_{p}(A ; b, a) .
\end{aligned}
$$

Using nearly identical steps we may also show that

LEMma 3.4. Given $A$ and $F$ in $\mathscr{W}_{p}([a, b])$, for each $\eta \geqslant \xi \in[a, b]$, the Stieltjes integral $\int_{\eta}^{\xi} d A F$ exists and satisfies, for any $\sigma \in[\eta, \xi]$,

$$
\left|\int_{\eta}^{\xi} d A F-d A(\xi, \eta) F(\sigma)\right| \leqslant 2\left[2+\zeta\left(\frac{2}{p}\right)\right] V_{p}(A ; \xi, \eta) V_{p}(F ; \xi, \eta) .
$$

Furthermore, as a function of $\xi, \int_{a}^{\xi} d A F$ belongs to $\mho_{p}([a, b])$.

4. The integral equation. In this section we shall use the Banach fixed point theorem to prove the existence of a unique solution to the evolution integral equation

$$
f(\xi)=x_{a}+\int_{a}^{\xi} d A f \quad(a \leqslant \xi \leqslant b)
$$

where $x_{a} \in X$ and $A \in \mathcal{U}_{p}([a, b])$. We shall need to keep in mind that for $[u, v] \subseteq$ $[a, b], W_{p}([u, v])$, when endowed with the norm

$$
\|f\|_{[u, v]}=\sup _{u \leqslant \theta \leqslant v}|f(\theta)|+V_{p}(f ; v, u),
$$

is a Banach space. The rather straightforward proof of this shall be omitted.

THEOREM 4.1. Given $A \in \mathcal{U}_{p}([a, b])$, for each $x_{a} \in X$ the integral equation (4.1) has a unique solution $f \in W_{p}([a, b])$.

Proof. It will suffice to show the existence of a positive number $\delta_{0}$ such that for each $\eta \in[a, b)$, if $I_{\eta}=\left[\eta, \min \left(\eta+\delta_{0}, b\right)\right]$ then the mapping $N: W_{p}\left(I_{\eta}\right) \rightarrow W_{p}\left(I_{\eta}\right)$ given by

$$
N f(\xi)=x_{a}+\int_{\eta}^{\xi} d A f, \quad \xi \in I_{\eta},
$$

is a contraction. Now from (3.5) we see that for $\delta \in(0, b-\eta]$,

$$
\begin{aligned}
V_{p}(N f-N g ; \eta+\delta, \eta) \leqslant & 2[2+\zeta(2 / p)] V_{p}(A ; \eta+\delta, \eta) V_{p}(f-g ; \eta+\delta, \eta) \\
& +\sup _{\theta \in[\eta, \eta+\delta]}|f(\theta)-g(\theta)| V_{p}(A ; \eta+\delta, \eta)
\end{aligned}
$$

and from (3.2) we have the bound

$$
\begin{aligned}
\sup _{\theta \in[\eta, \eta+\delta]}|N f(\theta)-N g(\theta)| \leqslant & 2[2+\zeta(2 / p)] V_{p}(A ; \eta+\delta, \eta) V_{p}(f-g ; \eta+\delta, \eta) \\
& +\sup _{\theta \in[\eta, \eta+\delta]}|f(\theta)-g(\theta)||A(\eta+\delta)-A(\eta)| .
\end{aligned}
$$


Next, using Lemma 2.4, choose $\delta_{0}>0$ such that for all $\mu \in[a, b)$ and all $0<\delta<$ $\min \left(\delta_{0}, b-\mu\right)$

$$
V_{p}(A ; \mu+\delta, \mu) \leqslant(4[2+\zeta(2 / p)])^{-1} .
$$

Then, from the preceding inequalities, we have, for all $f, g \in W_{p}([a, b])$,

$$
\|N f-N g\|_{I_{\eta}}<\frac{3}{4}\|f-g\|_{I_{\eta}} .
$$

The fact that $\mathcal{W}_{p}([a, b])$ is, like $W_{p}([a, b])$, also a Banach space under the corresponding norm allows for

TheOrem 4.2. Given $A \in \mathscr{W}_{p}([a, b])$, for each $P_{a} \in \mathscr{B} \mathcal{L}(X)$, the operator integral equation

$$
F(\xi)=P_{a}+\int_{a}^{\xi} d A F \quad(a \leqslant \xi \leqslant b)
$$

has a unique solution $F \in \mathcal{W}_{p}([a, b])$.

5. Existence of the product integral. The following lemma is of interest because $A$ is not assumed to be of bounded variation nor is $d A$ assumed to be dissipative.

LEMma 5.1. Given $A \in \mathcal{W}_{p}([a, b])$, there exists a constant $C$ such that if $[\mu, \nu] \subseteq$ $[a, b]$ and $\tau$ is any partition of $[\mu, \nu]$, then $\left|\Pi_{\tau}[I+d A]\right| \leqslant C$.

Proof. Given $\xi \in[a, b)$ let $\delta>0$ be such that $\xi+\delta<b$, and let $\sigma=\left\{\sigma_{i}\right\}_{0}^{n}$ be a partition of $[\xi, \xi+\delta]$. Then

$$
\begin{aligned}
\prod_{i=1}^{n}\left[I+d A\left(\sigma_{i}, \sigma_{i-1}\right)\right]= & I+\sum_{1 \leqslant i \leqslant n} d A\left(\sigma_{i}, \sigma_{i-1}\right)+\sum_{1 \leqslant j<i \leqslant n} d A\left(\sigma_{i}, \sigma_{i-1}\right) d A\left(\sigma_{j}, \sigma_{j-1}\right) \\
& +\sum_{1 \leqslant k<j<i \leqslant n} d A\left(\sigma_{i}, \sigma_{i-1}\right) d A\left(\sigma_{j}, \sigma_{j-1}\right) d A\left(\sigma_{k}, \sigma_{k-1}\right) \\
& +\cdots+d A\left(\sigma_{n}, \sigma_{n-1}\right) d A\left(\sigma_{n-1}, \sigma_{n-2}\right) \cdots d A\left(\sigma_{1}, \sigma_{0}\right) .
\end{aligned}
$$

Hence

$$
\prod_{\sigma}[I+d A]=\sum_{i=0}^{n} K_{i}(\xi+\delta),
$$

where for arbitrary $\sigma_{j} \in \sigma$ we have $K_{0}\left(\sigma_{j}\right) \equiv I$, and for $r=1,2, \ldots, n$, we have the recursive definition

$$
K_{r}\left(\sigma_{j}\right)=\sum_{i=1}^{j} d A\left(\sigma_{i}, \sigma_{i-1}\right) K_{r-1}\left(\sigma_{i-1}\right) .
$$

Our object shall be to find norm bounds on the $K_{r}(\xi+\delta)$ which are independent of $\sigma$ and summable. To this end, define, for $0 \leqslant r \leqslant n$,

$$
\alpha_{r}=\max _{1 \leqslant i \leqslant n}\left|K_{r}\left(\sigma_{i}\right)\right|
$$

and

$$
\beta_{r}=\max \left\{\left[\sum_{i=1}^{q}\left|K_{r}\left(\sigma_{u_{i}}\right)-K_{r}\left(\sigma_{u_{i-1}}\right)\right|^{p}\right]^{1 / p}: 0=u_{0}<u_{1}<\cdots<u_{q}=n\right\} .
$$


Now, for $0 \leqslant m \leqslant n-1$ and $\sigma_{k} \in \sigma$,

$$
\begin{aligned}
K_{r+1}\left(\sigma_{k}\right)-d A\left(\sigma_{k}, \xi\right) K_{r}\left(\sigma_{k}\right) & =\sum_{i=1}^{k} d A\left(\sigma_{i}, \sigma_{i-1}\right) K_{r}\left(\sigma_{i-1}\right)-d A\left(\sigma_{k}, \xi\right) K_{r}\left(\sigma_{k}\right) \\
& =-\sum_{1 \leqslant i \leqslant j \leqslant k} d A\left(\sigma_{i}, \sigma_{i-1}\right)\left(K_{r}\left(\sigma_{j}\right)-K_{r}\left(\sigma_{j-1}\right)\right) .
\end{aligned}
$$

Application of Lemma 3.2 therefore yields

$$
\alpha_{r+1} \leqslant[1+\zeta(2 / p)] V_{p}(A ; \xi+\delta, \xi) \beta_{r}+V_{p}(A ; \xi+\delta, \xi) \alpha_{r} .
$$

Next consider

$$
\begin{aligned}
K_{r+1}\left(\sigma_{u_{i}}\right)-K_{r+1}\left(\sigma_{u_{i-1}}\right)=\sum_{j=u_{i+1}}^{u_{i}} d A\left(\sigma_{j}, \sigma_{j-1}\right) K_{r}\left(\sigma_{j-1}\right) \\
=-\sum_{u_{i-1} \leqslant j \leqslant k \leqslant u_{i}} d A\left(\sigma_{j}, \sigma_{j-1}\right)\left(K_{r}\left(\sigma_{k}\right)-K_{r}\left(\sigma_{k-1}\right)\right)+d A\left(\sigma_{u_{i}}, \sigma_{u_{t-1}}\right) K_{r}\left(\sigma_{u_{i}}\right) .
\end{aligned}
$$

Applying first Minkowski's inequality and then Lemma 3.2 gives

$$
\begin{aligned}
\beta_{r+1} \leqslant & \max \left\{\sum_{i=1}^{q}\left|\sum_{u_{i-1} \leqslant j \leqslant k \leqslant u_{i}} d A\left(\sigma_{j}, \sigma_{j-1}\right) d K_{r}\left(\sigma_{k}, \sigma_{k-1}\right)\right|^{p}:\right. \\
& \left.0<u_{0}<\cdots<u_{q}=n\right\}^{1 / p} \\
& +\max \left\{\sum_{i=1}^{q}\left|d A\left(\sigma_{i}, \sigma_{i-1}\right)\right|^{p}\left|K_{r}\left(\sigma_{u_{i}}\right)\right|^{p}: 0<u_{0}<\cdots<u_{q}=n\right\}^{1 / p} \\
\leqslant & \max \left\{\sum_{i=1}^{q}\left[1+\zeta\left(\frac{2}{p}\right)\right]^{p} V_{p}^{p}\left(A ; \sigma_{u_{i}}, \sigma_{u_{i-1}}\right) \beta_{r}^{p}: 0<u_{0}<\cdots<u_{q}=n\right\}^{1 / p} \\
& +\max \left\{\sum_{i=1}^{q}\left|d A\left(\sigma_{u_{i}}, \sigma_{u_{i-1}}\right)\right|^{p}: 0<u_{0}<\cdots<u_{q}=n\right\}^{1 / p} \alpha_{r} \\
\leqslant & {[1+\zeta(2 / p)] V_{p}(A ; \xi+\delta, \xi) \beta_{r}+V_{p}(A ; \xi+\delta, \xi) \alpha_{r} . }
\end{aligned}
$$

Using matrix notation we may express (5.1) and (5.2) as

$$
\left(\begin{array}{c}
\alpha_{r+1} \\
\beta_{r+1}
\end{array}\right) \leqslant Q(\delta)\left(\begin{array}{c}
\alpha_{r} \\
\beta_{r}
\end{array}\right)
$$

where

$$
Q(\delta)=V_{p}(A ; \xi+\delta, \xi)\left(\begin{array}{ll}
1 & {[1+\zeta(2 / p)]} \\
1 & {[1+\zeta(2 / p)]}
\end{array}\right)
$$

It follows that, for all positive integers $r$,

$$
\left(\begin{array}{c}
\alpha_{r} \\
\beta_{r}
\end{array}\right) \leqslant Q^{r}(\delta)\left(\begin{array}{c}
\alpha_{0} \\
\beta_{0}
\end{array}\right)
$$


where $\alpha_{0}=1$ and $\beta_{0}=0$. Observe next that

$$
\left[\begin{array}{ll}
1 & 1 \\
1 & 1
\end{array}\right]^{r}=2^{r-1}\left[\begin{array}{ll}
1 & 1 \\
1 & 1
\end{array}\right]
$$

Thus (5.4) may be replaced by the estimate

$$
\left(\begin{array}{l}
\alpha_{r} \\
\beta_{r}
\end{array}\right) \leqslant\left(2\left[1+\zeta\left(\frac{2}{p}\right)\right] V_{p}(A ; \xi+\delta, \xi)\right)^{r}\left(\begin{array}{l}
1 \\
1
\end{array}\right) .
$$

Now let $\rho_{0}>0$ be such that, for all $s, t \in[a, b)$ with $0 \leqslant t-s \leqslant \rho_{0}, V_{p}(A ; t, s)<$ $(4[1+\zeta(2 / p)])^{-1}$. Hence if $\delta$ is chosen to be less than $\rho_{0}$, we have, from (5.5),

$$
\left|\prod_{\sigma}[I+d A]\right| \leqslant \sum_{i=0}^{n}\left|K_{i}(\xi+\delta)\right| \leqslant \sum_{i=0}^{n}\left(\frac{1}{2}\right)^{i} \leqslant 2,
$$

independent of $\xi$ or $\sigma$.

To conclude the proof, let $\tau=\left\{\tau_{i}\right\}_{0}^{m}$ be an arbitrary partition of the subinterval $[\mu, \nu] \subseteq[a, b]$. Let $\left\{\tau_{i_{r}}\right\}_{r \in g}$ be the set of all those partition points for which $\tau_{i_{r}}-\tau_{i_{r},} \geqslant \rho_{0}$, and let

$$
i_{*}= \begin{cases}\max \left\{i_{r}: i_{r} \in 9\right\} & \text { if } 9 \neq \varnothing, \\ 0 & \text { if } 9=\varnothing .\end{cases}
$$

It follows that

$$
\begin{aligned}
\left|\prod_{\tau}[I+d A]\right| \leqslant & \prod_{r \in g}\left|\left[I+d A\left(\tau_{i_{r}}, \tau_{i_{r-1}}\right)\right]\right| \\
& \times \prod_{r \in \mathcal{G}} \prod_{i_{r}<k<i_{r+1}-1}\left[I+d A\left(\tau_{k}, \tau_{k-1}\right)\right]|| \prod_{i_{*}<k \leqslant m}\left[I+d A\left(\tau_{k}, \tau_{k-1}\right)\right] \mid,
\end{aligned}
$$

where $\mathcal{G} \subseteq 9$ is the set of all $r$ such that $i_{r}+1<i_{r+1}$. Since $\mathscr{G}$ is finite, in fact

$$
|q| \leqslant \llbracket(b-a) / \rho_{0} \rrbracket+1 \equiv \mathfrak{p}_{0},
$$

a little thought reveals that the following bound holds:

$$
\left|\prod_{\tau}[I+d A]\right|<(1+\alpha)^{|\mathcal{|}|} 2^{\left(2 \mathfrak{p}_{0}+1\right)|g|} 2^{\left(2 \mathfrak{p}_{0}+1\right)},
$$

where $\alpha=\sup _{a \leqslant s \leqslant t \leqslant b}|d A(t, s)|$. Though surely this bound could be vastly strengthened, for our purpose, it will suffice to complete the proof.

TheOREM 5.1. Given $A \in \mathcal{W}_{p}([a, b])$ and $\xi \in[a, b]$, the product integral $\prod_{a}^{\xi}[I+d A]$ exists (in the operator-norm topology).

Proof. Let $F$ denote the solution to the integral equation of Theorem 4.2 when $P_{a}=I$, and let $\tau=\left\{\tau_{i}\right\}_{0}^{n}$ be a partition of $[a, \xi]$. Then for $1 \leqslant i \leqslant n$ we may write

$$
F\left(\tau_{i}\right)=\left[I+d A\left(\tau_{i}, \tau_{i-1}\right)\right] F\left(\tau_{i-1}\right)+\Delta_{i}
$$

where

$$
\Delta_{i}=\int_{\tau_{i-1}}^{\tau_{i}} d A F-d A\left(\tau_{i}, \tau_{i-1}\right) F\left(\tau_{i-1}\right)
$$


The solution to this difference equation is

$$
F\left(\tau_{j}\right)=\prod_{i=1}^{j}\left[I+d A\left(\tau_{i}, \tau_{i-1}\right)\right]+\sum_{i=1}^{j} \prod_{k=i+1}^{j}\left[I+d A\left(\tau_{k}, \tau_{k-1}\right)\right] \Delta_{i},
$$

for $j=1, \ldots, n$. In particular, we have, for $j=n$,

$$
\left|F(\xi)-\prod_{\tau}[I+d A]\right| \leqslant C \sum_{i=1}^{n}\left|\Delta_{i}\right|
$$

where the constant $C$ is taken from Lemma 5.1. Lemma 3.4 now provides

$$
\left|F(\xi)-\prod_{\tau}[I+d A]\right| \leqslant C^{\prime} \sum_{i=1}^{n} V_{p}\left(A ; \tau_{i}, \tau_{i-1}\right) V_{p}\left(F ; \tau_{i}, \tau_{i-1}\right),
$$

where $C^{\prime}=2[2+\zeta(2 / p)] C$. At this point the proof concludes as does the conclusion of Lemma 3.3; and as in that lemma, we have shown existence, beyond in the sense of successive refinements, in the more exacting Riemann sense.

Using a similar approach as above, we may also prove

THEOREM 5.2. If $f$ is the solution to the integral equation (4.1) then

$$
f(\xi)=\prod_{a}^{\xi}[I+d A] x_{a}
$$

Moreover, we have the

COROllary. The mapping $x_{a} \mapsto f(\xi)$ is a continuous mapping of $X$ into $W_{p}([a, b])$.

Proof. Let $g$ be the solution to (4.1) with the initial value $y_{a}$. Then $f(\xi)=F(\xi) x_{a}$ and $g(\xi)=F(\xi) y_{a}$, where $F(\xi)=\prod_{a}^{\xi}[I+d A]$. Hence,

$$
\begin{aligned}
\|f-g\|_{[a, b]} & =\sup _{\theta \in[a, b]}|f(\theta)-g(\theta)|+V_{p}(f-g ; b, a) \\
& \leqslant C\left|x_{a}-y_{a}\right|+V_{p}(F ; b, a)\left|x_{a}-y_{a}\right| . \quad \square
\end{aligned}
$$

REMARK 5.1. The method of Theorem 5.1 may also be used to prove that for any

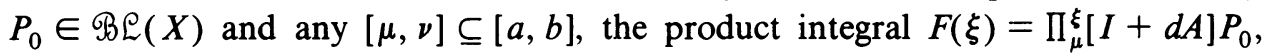
$\xi \in[\mu, \nu]$, exists as an element of $\mathscr{W}_{p}([a, b])$ and satisfies $F(\nu)=P_{0}+\int_{\mu}^{\nu} d A F$.

\section{A local time-ordered exponential.}

THEOREM 6.1. Given $A \in \mathcal{W}_{p}([a, b])$ there exists $\rho_{0}>0$ such that for every subinterval $[\mu, \nu] \subseteq[a, b]$ with $\nu-\mu<\rho_{0}$, the product integral $U(t, s)=\Pi_{s}^{t}[I+d A]$ has the series representation

$$
\prod_{s}^{t}[I+d A]=\sum_{k=0}^{\infty} H_{k}(t, s) \quad \forall s \leqslant t \in[\mu, \nu],
$$

where $H_{0}(t, s) \equiv I$ and, inductively,

$$
H_{n+1}(t, s)=\int_{s}^{t} d A H_{n}(\cdot, s), \quad n=0,1, \ldots
$$


Proof. Choose $\rho_{0}$ as in Lemma 5.1 so that if $a \leqslant s \leqslant t \leqslant b$ and $t-s<\rho_{0}$, then $4[2+\zeta(2 / p)] V_{p}(A ; t, s)<\frac{1}{2}$. Now each function $H_{n}(\xi, s), \xi \in[s, t]$, is in $\mathcal{Q}_{p}([s, t])$. Hence, should we define for $n=0,1, \ldots$,

$$
T_{n}(\xi)=U(\xi, s)-\sum_{k=0}^{n} H_{k}(\xi, s), \quad \xi \in[s, t],
$$

then each $T_{n}$ is in $\mathscr{W}_{p}([s, t])$. Furthermore, applying Theorem 4.2 and Remark 5.1 shows that

$$
\begin{aligned}
T_{n+1}(\xi) & =U(\xi, s)-\sum_{k=0}^{n+1} H_{k}(\xi, s)=I+\int_{s}^{\xi} d A U(\cdot, s)-\sum_{k=0}^{n}\left(\int_{s}^{\xi} d A H_{k}\right)-I \\
& =\int_{s}^{\xi} d A\left[U(\cdot, s)-\sum_{k=0}^{n} H_{k}\right]=\int_{s}^{\xi} d A T_{n}
\end{aligned}
$$

for all $\xi \in[s, t]$. Our goal shall be to prove that $T_{n}(t) \rightarrow 0$ as $n \rightarrow \infty$. Now define $\alpha_{n}=\max \left\{\left|T_{n}(\theta)\right|: s \leqslant \theta \leqslant t\right\}$ and $\beta_{n}=V_{p}\left(T_{n} ; t, s\right)$. Thus, since for $\theta \in[s, t]$,

$$
\left|T_{n+1}(\theta)\right| \leqslant\left|\int_{s}^{\theta} d A T_{n}-d A(\theta, s) T_{n}(\theta)\right|+|d A(\theta, s)|\left|T_{n}(\theta)\right|,
$$

we have

$$
\alpha_{n+1} \leqslant 2[2+\zeta(2 / p)] V_{p}(A ; t, s) \beta_{n}+V_{p}(A ; t, s) \alpha_{n} .
$$

As well, if $\tau=\left\{\tau_{i}\right\}_{0}^{n}$ is a partition of $[s, t]$ then

$$
\begin{gathered}
{\left[\sum_{\tau}\left|d T_{n+1}\right|^{p}\right]^{1 / p} \leqslant\left[\sum_{i=1}^{n}\left\{\left|\int_{\tau_{i-1}}^{\tau_{i}} d A T_{n}-d A\left(\tau_{i}, \tau_{i-1}\right) T_{n}\left(\tau_{i}\right)\right|+\left|d A\left(\tau_{i}, \tau_{i-1}\right) T_{n}\left(\tau_{i}\right)\right|\right\}^{p}\right]^{1 / p}} \\
\leqslant\left[\sum_{i=1}^{n}\left(2\left[2+\zeta\left(\frac{2}{p}\right)\right]\right)^{p} V_{p}^{p}\left(A ; \tau_{i}, \tau_{i-1}\right) V_{p}^{p}\left(T_{n} ; \tau_{i}, \tau_{i-1}\right)\right]^{1 / p}+V_{p}(A ; t, s) \alpha_{n} .
\end{gathered}
$$

Hence,

$$
\beta_{n+1} \leqslant 2[2+\zeta(2 / p)] V_{p}(A ; t, s) \beta_{n}+V_{p}(A ; t, s) \alpha_{n} .
$$

Now, just as in the proof of Lemma 5.1, inequalities (6.1) and (6.2) imply that

$$
\alpha_{n} \leqslant\left(4[2+\zeta(2 / p)] V_{p}(A ; t, s)\right)^{n} \leqslant(1 / 2)^{n} \rightarrow 0 \quad \text { as } n \rightarrow \infty
$$

7. Existence of the sum integral. We define the evolution class $\mathcal{E}_{p}([a, b])$ to be the set of all evolutions $U(\cdot)$ continuous in the operator norm topology and such that the $p$-variation of the interval function $U-I$ over $[a, b]$,

$$
V_{p}(U-I ; b, a)=\sup \left\{\left[\sum_{\tau}|U-I|^{p}\right]^{1 / p}: \tau \text { is a partition of }[a, b]\right\},
$$

is finite. Equivalently, it is easily shown that $\mathcal{E}_{p}([a, b])$ is the set of all evolutions $U$ such that either $U(\cdot, a)$ or $U(b, \cdot)$ belongs to ${ }_{0}([a, b])$. 
LEMMA 7.1. If $U \in \mathcal{E}_{p}([a, b])$ and for each $\mu \in[a, b)$ we define $G_{\mu}(\xi)=U(\xi, \mu)$, $\xi \in[\mu, b]$, then $G_{\mu} \in \mathcal{O} \int_{p}([\mu, b])$, and for all $\nu \in[\mu, b]$,

$$
V_{p}\left(G_{\mu} ; \nu, \mu\right) \leqslant \sup _{a \leqslant s \leqslant t \leqslant b}|U(t, s)| V_{p}(U-I ; \nu, \mu) .
$$

Proof. The proof is immediate from the identity

$$
G_{\mu}(t)-G_{\mu}(s)=[U(t, s)-I] G_{\mu}(s) \quad(u \leqslant s \leqslant t \leqslant b)
$$

and the fact that $U(\cdot)$ is continuous.

Lemma 7.2. There exists $\delta_{0}>0$ independent of $\mu \in[a, b)$ such that

$$
\left|G_{\mu}(t)-I\right|<\frac{1}{2} \quad \text { for all } \mu \leqslant t \leqslant \min \left(\mu+\delta_{0}, b\right) .
$$

Hence $G_{\mu}(\cdot)$ is invertible on the interval $\left[\mu, \min \left(\mu+\delta_{0}, b\right)\right]$.

Proof. The proof follows directly from the fact that $U(\cdot)$ is continuous on a compact domain and $U(t, t) \equiv I$.

Lemma 7.3. With $\delta_{0}$ as in Lemma 7.2, for all $\mu \in[a, b)$ and all $\delta \in\left[0, \delta_{0}\right]$ such that $\mu+\delta \leqslant b$,

$$
G_{\mu}^{-1} \in \mathscr{Q} \int_{p}([\mu+\delta, \mu])
$$

and

$$
V_{p}\left(G_{\mu}^{-1} ; \mu+\delta, \mu\right) \leqslant 4 \sup _{a \leqslant s \leqslant t \leqslant b}|U(t, s)| V_{p}(U-I ; \mu+\delta, \mu) .
$$

Proof. The Neumann series for $G_{\mu}^{-1}$ provides the bound

$$
\left|G_{\mu}^{-1}(t)\right| \leqslant 1 /\left(1-\left|G_{\mu}(t)-I\right|\right) .
$$

Hence $G_{\mu}^{-1}$ is uniformly bounded (by 2 ) on $[\mu, \mu+\delta]$. The proof now follows from the identity

$$
G_{\mu}^{-1}(t)-G_{\mu}^{-1}(s)=G_{\mu}^{-1}(s)\left[G_{\mu}(s)-G_{\mu}(t)\right] G_{\mu}^{-1}(t) .
$$

TheOREM 7.1. For each $\xi \in[a, b]$, the sum integral $\Sigma_{a}^{\xi}[U-I]$ exists and, as a function of $\xi$, defines a member of $\mho_{p}([a, b])$.

Proof. Divide $[a, \xi]$ into subintervals $\left[a, a+\delta_{0}\right],\left[a+\delta_{0}, a+2 \delta_{0}\right], \ldots,[a+$ $\left.k \delta_{0}, \xi\right]$ where $k$ satisfies $0 \leqslant \xi-a-k \delta_{0} \leqslant \delta_{0}$. Using (7.1) we see that on the first $k-1$ subintervals, the sum integral $\Sigma[U-I]$ exists as

$$
\int_{a+j \delta_{0}}^{a+(j+1) \delta_{0}} d G_{a+j \delta_{0}} G_{a+j \delta_{0}}^{-1} \quad(j=1, \ldots, k-1),
$$

and on the last subinterval as

$$
\int_{a+k \delta_{0}}^{\xi} d G_{a+k \delta_{0}} G_{a+k \delta_{0}}^{-1}
$$

Hence the sum integral exists on the full interval $[a, \xi]$ and, by Lemma 3.4, defines an element of $\mathcal{Q}_{p}([a, \xi])$.

We may also prove the companion

Theorem 7.2 If $A \in \mathcal{U}_{p}([a, b])$ and $U=\Pi[I+d A]$, then $U \in \mathcal{E}_{p}([a, b])$. 


\section{The evolution representation.}

THEOREM 8.1. For every $U \in \mathcal{E}_{p}([a, b])$ there exists a generator $A \in \mathcal{Q}_{p}([a, b])$, unique up to additive constant operator, such that $U(t, s)=\prod_{s}^{t}[I+d A]$ for all $s \leqslant t \in[a, b]$.

Proof.The generator $A$ is obtained as the sum integral $A(\xi)=\sum_{a}^{\xi}[U-I], \xi \in$ $[a, b]$. First we show that $U$ can be recovered from the product integral $\Pi[I+d A]$, whose existence is established by Theorems 5.1 and 7.1, and reestablished by different means in what follows. Given $s \leqslant t \in[a, b]$, let $\tau=\left\{\tau_{i}\right\}_{0}^{n}$ be a partition of $[s, t]$ with $|\tau|$ no greater than the $\delta_{0}$ of Lemma 7.2. There exists a constant $\Gamma$ such that

$$
\begin{aligned}
\mid \prod_{\tau}[I+ & d A]-U(t, s) \mid \\
& =\left|\sum_{i=1}^{n} U\left(t, \tau_{i}\right)\left[I+d A\left(\tau_{i}, \tau_{i-1}\right)-U\left(\tau_{i}, \tau_{i-1}\right)\right] \prod_{j=1}^{i-1}\left[I+d A\left(\tau_{j}, \tau_{j-1}\right)\right]\right| \\
& \leqslant \Gamma \sum_{i=1}^{n}\left|d A\left(\tau_{i}, \tau_{i-1}\right)-\left(U\left(\tau_{i}, \tau_{i-1}\right)-I\right)\right| .
\end{aligned}
$$

With $G_{\tau_{k}}(\cdot)$, as in the proof of Theorem 7.1, equal to $U\left(\cdot, \tau_{k}\right)$ and $\tau_{i}-\tau_{i-1} \leqslant \delta_{0}$, the preceding sum is equal to

$$
\begin{aligned}
\Gamma \sum_{i=1}^{n} \mid \int_{\tau_{i-1}}^{\tau_{i}} d G_{\tau_{i-1}} G_{\tau_{i, 1}}^{-1} & -\left[G_{\tau_{i}, 1}\left(\tau_{i}\right)-G_{\tau_{i-1}}\left(\tau_{i-1}\right)\right] G_{\tau_{i}, 1}^{-1}\left(\tau_{i-1}\right) \mid \\
& \leqslant \Gamma \sum_{i=1}^{n} 2\left[2+\zeta\left(\frac{2}{p}\right)\right] V_{p}\left(G_{\tau_{i-1}} ; \tau_{i}, \tau_{i-1}\right) V_{p}\left(G_{\tau_{i-1}}^{-1} ; \tau_{i}, \tau_{i-1}\right) .
\end{aligned}
$$

Now by Lemmas 7.2 and 7.3 there exists a constant $\Gamma^{\prime}$ such that this latter sum is bounded above by

$$
\Gamma^{\prime} \sum_{i=1}^{n} V_{p}^{2}\left(U-I ; \tau_{i}, \tau_{i-1}\right) \leqslant \Gamma^{\prime} \max _{1 \leqslant i \leqslant n} V_{p}^{2-p}\left(U-I ; \tau_{i}, \tau_{i-1}\right) \sum_{i=1}^{n} V_{p}^{p}\left(U-I ; \tau_{i}, \tau_{i-1}\right)
$$

The fact that Lemma 2.4 and its proof are also true when $A$ is replaced by $U-I$, together with the superadditivity of $V_{p}^{p}(U-I ; \cdot)$, gives that for $|\tau|$ sufficiently small, the above sum can be made arbitrarily small. Thus we have

$$
U(t, s)=\prod_{s}^{t}[I+d A] .
$$

As for the uniqueness of $A$, suppose that $B \in \mathcal{W}_{p}([a, b])$ also satisfies $\prod_{s}^{t}[I+d B]$ $=U(t, s)$ for all $s \leqslant t \in[a, b]$. We shall show that $d B(\nu, \mu)$ equals $d A(\nu, \mu)$ for every $\mu \leqslant \nu \in[a, b]$. Now given $\varepsilon>0$, let $\tau=\left\{\tau_{i}\right\}_{0}^{n}$ be a partition of $[\mu, \nu]$ such that

$$
\left|d A(\nu, \mu)-\sum_{\tau}[U-I]\right| \leqslant \frac{\varepsilon}{2},
$$


and for all $\tau_{i} \in \tau$,

$$
\begin{gathered}
V_{p}^{2-p}\left(B ; \tau_{i}, \tau_{i-1}\right)<\frac{\varepsilon}{4}\left(\left[2+\zeta\left(\frac{2}{p}\right)\right] \sup _{s \leqslant t \in[a, b]}|U(t, s)| V_{p}^{p}(B ; \nu, \mu)\right)^{-1}, \\
V_{p}^{2-p}\left(U-I ; \tau_{i}, \tau_{i-1}\right)<\frac{\varepsilon}{4}\left(\left[2+\zeta\left(\frac{2}{p}\right)\right] \sup _{s \leqslant t \in[a, b]}|U(t, s)| V_{p}^{p}(U-I ; \nu, \mu)\right)^{-1} .
\end{gathered}
$$

Then, using Lemmas 3.4 and 7.1 and Remark 5.1, we have

$$
\begin{aligned}
\mid d B & (\nu, \mu)-d A(\nu, \mu)|\leqslant| d B(\nu, \mu)-\sum_{\tau}[U-I] \mid+\frac{\varepsilon}{2} \\
& =\left|\sum_{i=1}^{n}\left\{d B\left(\tau_{i}, \tau_{i-1}\right)-\left[U\left(\tau_{i}, \tau_{i-1}\right)-I\right]\right\}\right|+\frac{\varepsilon}{2} \\
& =\left|\sum_{i=1}^{n}\left\{\int_{\tau_{i-1}}^{\tau_{i}} d B U\left(\cdot, \tau_{i-1}\right)-d B\left(\tau_{i}, \tau_{i-1}\right) U\left(\tau_{i-1}, \tau_{i-1}\right)\right\}\right|+\frac{\varepsilon}{2} \\
& \leqslant \sum_{i=1}^{n} 2\left[2+\zeta\left(\frac{2}{p}\right)\right] V_{p}\left(B ; \tau_{i}, \tau_{i-1}\right) V_{p}\left(U\left(\cdot, \tau_{i-1}\right) ; \tau_{i}, \tau_{i-1}\right)+\frac{\varepsilon}{2} \\
& \leqslant \sum_{i=1}^{n} 2\left[2+\zeta\left(\frac{2}{p}\right)\right]_{s<t \in[a, b]} \sup _{i}|U(t, s)| V_{p}\left(B ; \tau_{i}, \tau_{i-1}\right) V_{p}\left(U-I ; \tau_{i}, \tau_{i-1}\right)+\frac{\varepsilon}{2} \\
& \leqslant\left[2+\zeta\left(\frac{2}{p}\right) \sup _{s \leqslant t([a, b])}|U(t, s)| \sum_{i=1}^{n}\left(V_{p}^{2}\left(B, \tau_{i}, \tau_{i-1}\right)\right.\right. \\
& \leqslant \frac{\varepsilon}{4} \sum_{i=1}^{n}\left\{\frac{V_{p}^{p}\left(B ; \tau_{i}, \tau_{i-1}\right)}{V_{p}^{p}(B ; \nu, \mu)}+\frac{V_{p}^{p}\left(U-I ; \tau_{i}, \tau_{i-1}\right)}{V_{p}^{p}(U-I ; \nu, \mu)}+\frac{\varepsilon}{2} \leqslant \varepsilon .\right.
\end{aligned}
$$

\section{REFERENCES}

1. A. L. Cauchy, Cours d'analyse de l'Ecole Royal Polytechnique, 1821.

2. J. C. Burkill and F. W. Gehring, A scale of integrals from Lebesgue's to Denjoy's, Quart. J. Math. Oxford Ser. (2) 4 (1953), 210-20.

3. M. Bruneau, Variation totale d'une fonction, Springer-Verlag, Berlin, 1974.

4. M. A. Freedman, A faithful Hille-Yosida theorem for finite dimensional evolutions, Trans. Amer. Math. Soc. 265 (1981), 563-573.

5 . , Necessary and sufficient conditions for discontinuous evolutions with applications to Stieltjes integral equations, J. Integral Equations (to appear).

6. F. W. Gehring, A study of $\alpha$-variation, Trans. Amer. Math. Soc. 76 (1954), 420-443.

7. J. V. Herod and R. W. McKelvey, A Hille-Yosida theory for evolutions, Israel J. Math. 36 (1980), 13-40.

8. E. R. Love, A generalization of absolute continuity, J. London Math. Soc. 26 (1951), 1-13.

9. J. S. Mac Nerney, Integral equations and semigroups, Illinois J. Math. 7 (1963), 148-173.

10. __ A linear initial-value problem, Bull. Amer. Math. Soc. 69 (1963), 314-329.

11. D. S. Nathan, One-parameter groups of transformations in abstract vector spaces, Duke Math. J. 1 (1935), 518-526.

12. N. Wiener, The quadratic variation of a function and its Fourier coefficients, J. Math. Phys. Sci. 3 (1924), 73-94.

13. L. C. Young, An inequality of the Hölder type, connected with Stieltjes integration, Acta Math. 67 (1936), 251-282. 